\title{
Functional morphology and adaptive significance of the mucous membrane of the corners of the mouth in ruminants
}

\author{
Raisa Khatsaeva $^{1, *}$, Soslan Kozyrev², and Arslan Khasaev ${ }^{3}$ \\ ${ }^{1}$ Institute of Ecology and Evolution named after A.N. Severtsov RAS, 119570, 33, Leninsky av., \\ Moscow, Russian Federation \\ ${ }^{2}$ North Caucasian Research Institute of Mining and Piedmont Agriculture - branch of the Federal \\ State Budgetary Scientific Institution Federal Scientific Center Vladikavkaz Scientific Center of the \\ Russian Academy of Sciences, 363110, 1 settlement Mikhailovskoe Williams str., Vladikavkaz, \\ Russian Federation \\ ${ }^{3}$ Dagestan State Agrarian University named after M.M. Dzhambulatova, 367032, 180, M. Gaszhieva \\ str., Makhachkala, Russian Federation
}

\begin{abstract}
Using the methods of scanning electron microscopy, morphological and functional features of the mucous membrane of the corners of the mouth in representatives of Bovinae cattle [Bos taurus taurus] with normal and insufficient nutrition and their adaptive significance were revealed. Determination of the morphology of the mucous membrane and its functional structures of the corners of the mouth of ruminants by the specificity of their nutrition and physical condition is shown. Compensatory mechanisms have been identified in the mucous membrane of the corners of the mouth in Bos taurus taurus, caused by poor-quality malnutrition. The ultrastructural features of the architectonics of the mucous membrane and its functional structures of the corners of the mouth and microbiome were revealed in connection with the specific nutritional characteristics of the compared animals. The obtained information can be used in development of the foundations of rational feeding of ruminants, including keeping wild species in captivity and solving the problems of rational nature management and conservation of biodiversity.
\end{abstract}

\section{Introduction}

Adaptation to herbivorousness has played a significant role in the evolution of mammals. It determined their ecological, behavioral and morphophysiological adaptations. Obviously, the most important manifestations of adaptations were morphological transformations of the digestive system and the use of external suppliers of enzymes symbionts. To date, a lot of scientific data on the comparative morphology of the digestive tract of ruminants have been accumulated, allowing to judge the relationship of the morphology of the digestive tract with the nature of nutrition and vital activity of symbionts

\footnotetext{
* Corresponding author: r.khatsaeva@mail.ru
} 
[1], [2], [3]. There are papers to identify morphofunctional manifestations of adaptations of the gastrointestinal tract to the nature of food and ecology [4], [5], [6], [7], [8], [9]. All comparative studies of food adaptations concern mainly the gastrointestinal tract [10], [11], [12], [13]. Until now, there are no studies on a comprehensive study of the digestive organs, starting with the oral cavity. The morphological and functional structures of the mucous membrane of the oral cavity of ruminants - the papillae of the corners of the mouth, which play a significant role in the processes of obtaining and processing plant food in ruminants - have not been studied at all, there is no description of the oral microbiome, and its role in the processes of digestion and formation of food specialization. In the selection of ruminants, the achievement of programmable results is used in order to combine the productivity potential with adaptation to the conditions of industrial technologies, and any trait can be selected as a target [14], [15], [16], [17]. All this indicates the growing importance of studies of the morphophysiological adaptive characteristics of the breeding material. Based on the foregoing, the study of the morphofunctional features of the mucous membrane of the oral cavity of ruminants and the microbiome on its surface, in connection with the specifics of nutrition, has undoubted theoretical and practical importance.

The aim of this study was to study the microscopic structure of the mucous membrane, its morphofunctional structures and the microbiome in the corners of the mouth in adults of Bos taurus taurus with normal and improper nutrition.

\section{Methods}

The corners of the mouth of two groups of adult Bos taurus taurus with different conditions of keeping and feeding were used as objects of the study. The first group - two animals with pasture housing, sufficient nutrition with natural grazing of herbaceous vegetation and the second - two animals with domestic maintenance and improper nutrition when using hot waste from the production of ethyl alcohol. The material was mined in the Republic of Kabardino-Balkaria in 2019. All animals were slaughtered for economic reasons.

The study used methods of scanning electron microscopy. For electron microscopic studies, objects (pieces of the corners of the mouth) were fixed in $10 \%$ formalin. After fixation, the samples were washed in water, then passed through a series of ethyl alcohol of ascending concentration (from $30 \%$ to $100 \%$ ) and acetone. After that, the samples were dried in an automatic drying unit at the critical point Leica EM CPD300 (Leica Microsystems Vertrieb $\mathrm{GmbH}$, Austria). To ensure the conductivity of the samples under study, they were deposited with gold on Q150R ES Plus equipment (Quorum Technologies Ltd, United Kingdom). The study and photography of the samples under study was carried out using a scanning electron microscope - TESCAN MIRA3 LMH (Brno, s.r.o., Czech Republic).

\section{Results}

The mucous membrane of the cheeks in the area of the corners of the mouth in the animals we compared with normal and inadequate nutrition had a different structure (Fig. 1 A, B). In healthy animals with a normal diet, the structure of the papillae was typical of ruminants. Buccal papillae, were lined with stratified squamous keratinizing epithelium and directed back towards the pharynx, the highest and densely located, were located in the area from the corner of the mouth to the beginning of the molars, the structure of the papillae was unevenly short, long, with a wide or narrow base, with a smooth surface, or with longitudinal grooves. 
The papillae were located unidirectionally, had various base lengths and widths, an almost smooth surface relief with pointed or blunt tips (Fig. 1A). In animals with insufficient nutrition, the papillae were curved, had a wrinkled appearance, their surface with numerous waves and influxes, with multiple splits and chips on the sides and at the tips (Fig. 1. B).

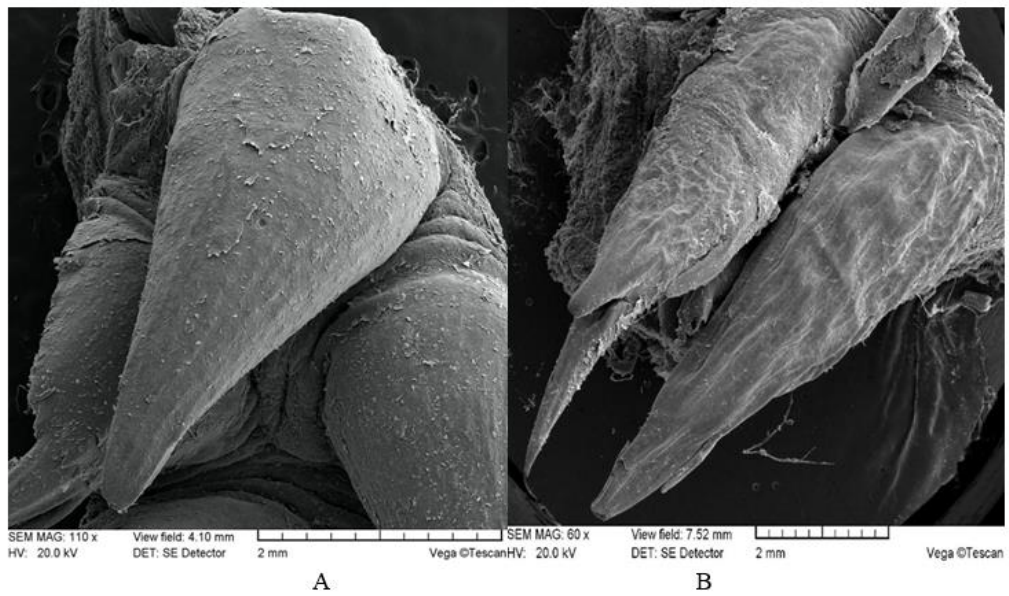

Fig. 1. General view of the papillae of the mucous membrane of the corners of the mouth of Bos taurus taurus . A - with a normal diet, B - with an unhealthy diet.

The tips of the papillae of the mucous membrane of the corners of the mouth in the compared animals had different degrees of wear. The first group had less wear than the second one (Fig. 1, 2. A, B).

In animals with normal nutrition, the cells of the superficial epithelium are arranged loosely and multilayered, with a slight degree of wear and a moderate number of peeling cells (Fig. 2A). In malnourished animals, the degree of desquamation was high, many with cut ends, and a large number of exfoliating cells on the surface (Fig. 2B).

The surface of the epithelium in the middle of the papillae in the compared animals also had a different structure (Fig. 3 A, B). In animals with a normal diet, it is smoother with a reticular structure. The cells were of unequal size and shape, mostly multifaceted. On the surface of the epithelium, there were many symbionts of various shapes located singly and in groups (Fig. 3A). In animals with insufficient nutrition, the surface of the middle of the papillae was wavy, deformed, with exfoliating cells. On the surface of the epithelium, there were a large number of crystals of various sizes and shapes and few symbionts (Fig. 3. B). 


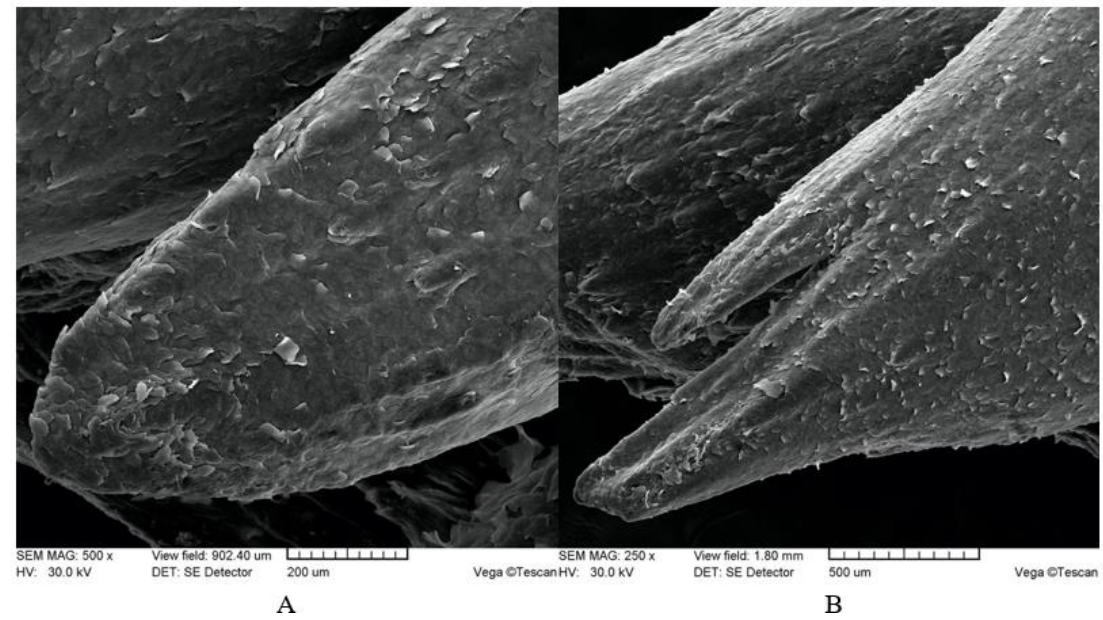

Fig.2. The surface structure of the epithelium of the tip of the papilla of the mucous membrane of the corners of the mouth of Bos taurus taurus . A - with normal nutrition, B - with insufficient nutrition.

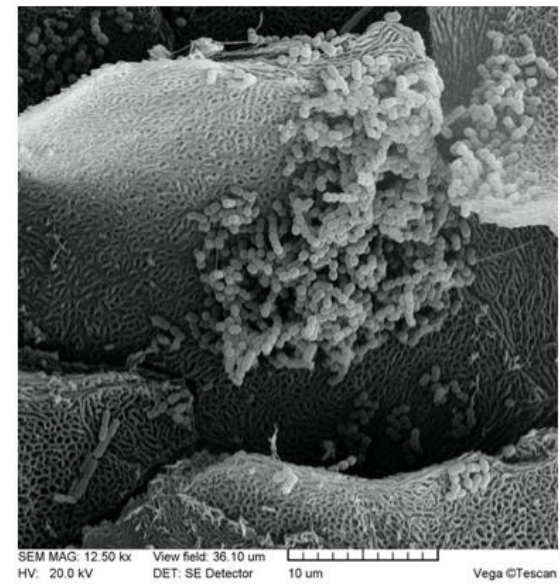

A

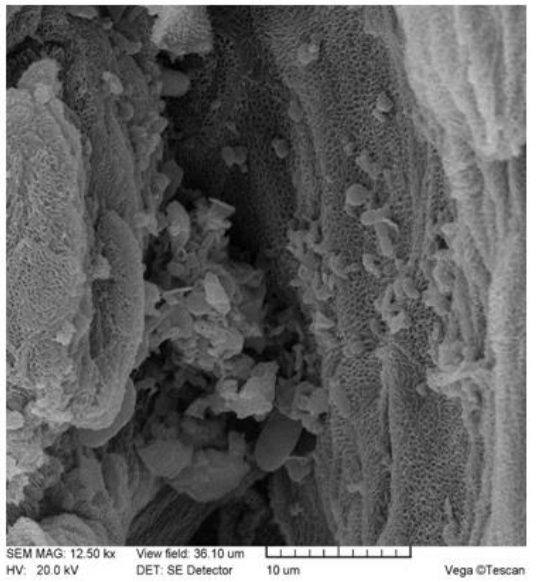

B

Fig. 3. The structure of the surface of the epithelium of the middle of the papilla of the mucous membrane of the corners of the mouth of Bos taurus taurus. A - with normal nutrition, B - with insufficient nutrition.

The epithelial surface of the base of the papillae of the corners of the mouth in the compared animals also has significant differences (Fig. 4 A, B). In an animal with a normal diet, the surface of the epithelium is smoother, with the exception of sloughing cells with a large number of different populations of symbionts (Fig. 4.A). In an animal with insufficient nutrition, the surface of the epithelium is wrinkled, forming wavy folds, on which single symbionts and crystals are located (Fig. 4 B). 


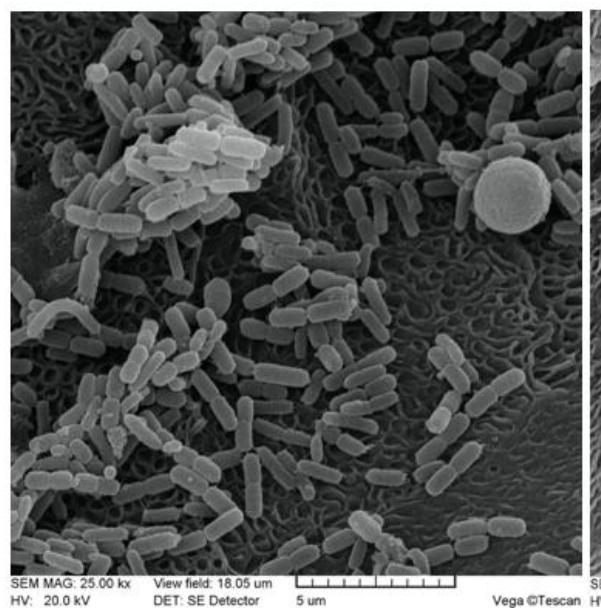

A

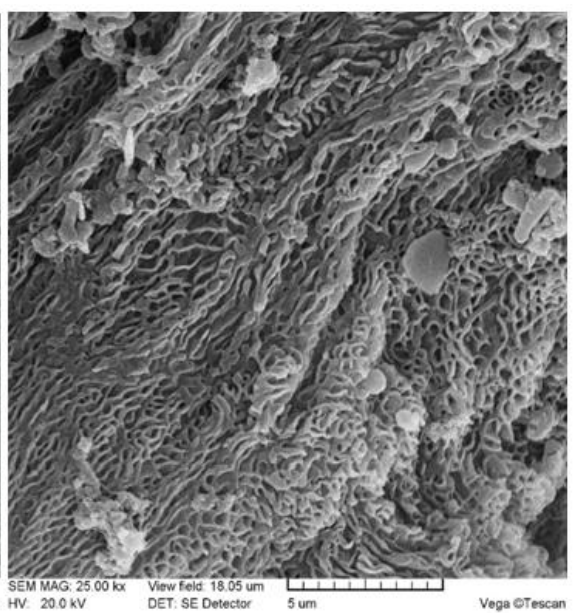

B

Fig. 4. Architectonics of the surface of the epithelium of the base of the papilla of the mucous membrane of the corners of the mouth of Bos taurus taurus. A - with normal nutrition, B with insufficient nutrition.

The surface of the epithelium of the mucous membrane between the papillae in the compared animals also had a different structure (Fig. 5 A, B). In animals of the first group with normal nutrition, the surface of the mucous membrane between the papillae was uneven with small depressions and had a very prominent reticular structure with a large number of different populations of symbionts and a small amount of mucus. In some cases, a very relief architecture of the surface of the epithelium with large slit-like depressions filled with significant accumulations of symbionts of different populations was observed (Fig. 5A). In animals of the second group with insufficient nutrition, the surface of the mucous membrane between the papillae had a wavy structure with large depressions, the reticulation of the ball was indistinct due to the large amount of mucus, which in places formed large accumulations, including around single symbionts (Fig. 5B).

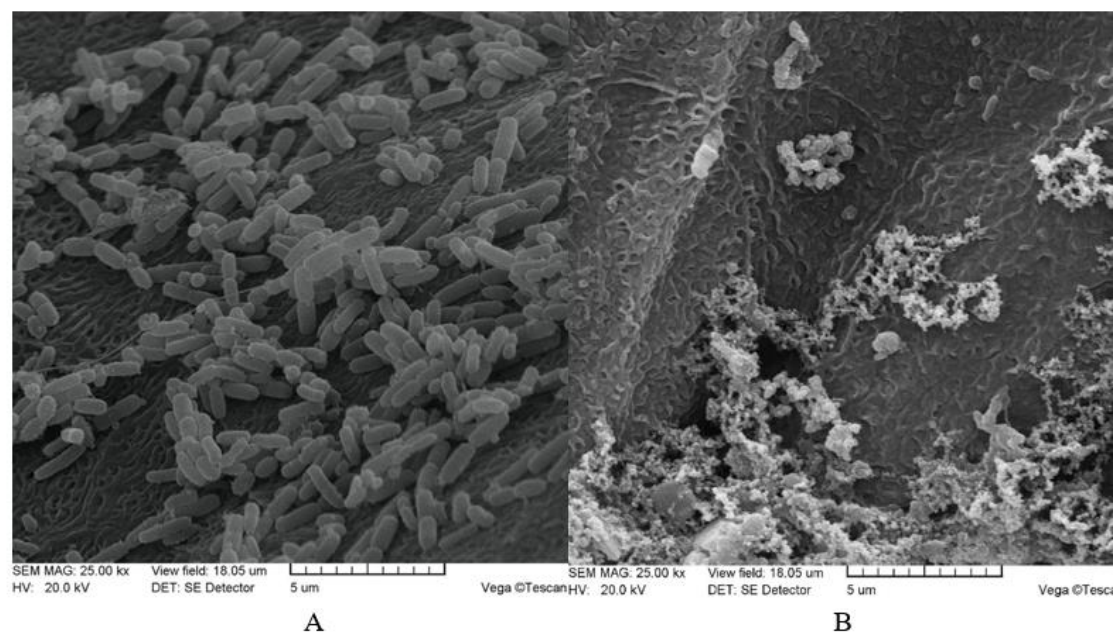

Fig.5. Symbionts on the surface of the epithelium between the papillae of the mucous membrane of the corner of the mouth of Bos taurus taurus. A - with normal nutrition, B - with insufficient nutrition. 


\section{Discussion}

Our studies have reliably revealed the presence in both groups of animals, characteristic of ruminant ungulates, the morphology of the mucous membrane of the corners of the mouth. The mucous membrane had a general structure plan. Cheek papillae, were lined with stratified squamous keratinizing epithelium and directed back towards the pharynx, the highest and densely located, were located in the area from the corner of the mouth to the beginning of the molars, the structure of the papillae was unevenly short, long, with a wide or narrow base, with a smooth surface, or with longitudinal grooves, with a sharp tip, or blunt, with clefts at the apex or on the side.

At the same time, significant features of the ultrafine structure of morphofunctional structures, the architectonics of the surface of the papillary epithelium, microbiota and its localization were revealed. There was a significant difference in the architectonics of the surface of the papillae, especially their tips (Fig. 1, 2 A, B).

In the animals of the first group, feeding on pasture vegetation, the papillae were intact with a relatively flat surface with evenly exfoliating cells, which is the norm (Fig. 1, 2 A). The surface of the epithelium of the mucous membrane of the corners of the mouth in the bulls of the first group had a distinct reticular structure with identical cells (Fig. 3A). The reticular structure contributes to an increase in the functional surface area and creates appropriate niches for symbiocenoses. The cells are basically the same size, multifaceted in shape. A large number of various symbionts are located on the surface of the epithelium (Fig. 3A).

In animals of the second group, the papillae were deformed, with splits at the tips and sides, the surface of the papillae was worn, with large and uneven areas of exfoliation with ulceration and holes (Fig. 1, 2 B). The surface of the epithelium of the mucous membrane of the corners of the mouth in the gobies of the second group had a wavy, indistinct structure with sharply delineated uneven cell boundaries (Fig. 2B). Mesh structure of the surface with unequal cells with stuck together edges. There is a lot of mucus on the surface of the cells, apparently having a protective function, and isolated microbes were found (Fig. 3B).

The morphological features of the fine structure of the surface of the papillae, which represent an ecological niche for symbiocenoses, determines the peculiarities of their localization, size, number, methods of attachment (Fig. 3 A, B). According to the surface topography, scattered single coccoid bacteria on a smoother surface or long chain coccoid bacteria in depressions with small cocci and rod-shaped forms located around it were located on it (Fig. 3, 4, 5 A, B).

In the first group of animals, on papillae with a more prominent epithelial surface, there were places completely covered with large accumulations of bacteria, mainly of one species - either coccoid or rod-shaped. At the same time, some bacteria lay freely, having no connections with each other and without mucus around, while others were connected to each other with the help of thin threads and with a small amount of mucus, or there were accumulations of bacteria from different species at the same time (Fig. 3, 4, 5 A). In some cases, a very relief architecture of the surface of the epithelium of the mucous membrane of the papillae with large slit-like depressions filled with significant accumulations of symbionts of different populations was observed (Fig. 3, 4 A). There were joint populations of coccoid and rod-shaped bacteria. In some cases, cocci were represented by single and double forms with a rough surface together with rod-shaped forms. In others, single and double cocci with a smooth surface and connecting filaments were observed in the vicinity of rod-shaped bacteria (Fig. 3A). All this diversity of symbionts, as well as their localization, corresponds to the norm for ruminant herbivorous animals. 
In the second group of animals on the surface of the epithelium of the papillae practically no symbionts were observed, with the exception of single small forms (Fig. 3, 4, $5 \mathrm{~B}$ ). In areas with a smoother surface topography, very small, enclosed in cells of the surface mesh structure, as well as numerous crystals of various shapes and sizes were observed (Figs. 3, 4, 5 B). In some cases, in the second study group of animals, chipped intact areas of the superficial epithelium were visible on the papillae, which also contained crystals and single small symbionts (Fig. 3, 4, 5 B). Perhaps the absence of symbiocenoses is also associated with the consumption of hot food, in which they died and only the smallest forms remained, inside the cells of the reticular structure of the surface of the mucous membrane. It can be assumed that a large number of different crystals on the surface of the mucous membrane is associated with some kind of protective reaction to hot food, which requires further verification.

The revealed morphological features of the mucous membrane of the corners of the mouth of Bos taurus taurus with normal and inadequate nutrition at the electronic microscopic level indicate adaptive mechanisms for changes caused by the specific nutritional status of ruminants. Wherein, these data testify to the limited adaptive capabilities when the feeding range of ruminants changes and can serve as a criterion in predicting the state of populations based on an assessment of their food base. In addition, the revealed features of the mucous membrane of the corner of the mouth of Bos taurus taurus in connection with the specifics of nutrition can be taken into account when developing the foundations of rational feeding during their selection, breeding and maintenance. All of the above demonstrates the determinism of the morphological features of the mucous membrane of the oral cavity of ruminants by the specificity of their nutrition.

\section{Conclusion}

Therefore, the results of our comparative studies of the morphology of the papillae of the oral mucosa in bulls (Bos taurus taurus), under different conditions of maintenance and nutrition, at the electronic microscopic level, revealed a general plan of their structure, as well as features reflecting the specificity of nutrition.

In the first group of animals, feeding on pasture vegetation, the morphology of the mucous membrane of the corners of the mouth was intact, the papillae were intact, with evenly peeling cells, which is the norm. The surface of the epithelium of the mucous membrane of the corners of the mouth in the bulls of the first group had a clearly expressed reticular structure with the same polyhedral cells. On the surface of the mucous membrane, there was a large number of various microflora, localized according to the architectonics of the surface of the epithelium. Obviously, in the first group, the morphology of functional structures and the state of symbiocenoses corresponded to the norm, which reflects their ecological adaptability to the assimilation of coarse herbaceous fodder characteristic of ruminants.

The features identified in the second group, kept at home and ate hot food, can be considered violations of the ultrastructure of the oral mucosa and its functional structures papillae, almost complete absence of symbiocenoses, with the exception of very small forms and a large number of various crystals and mucus.

The revealed morphological features of the mucous membrane of the corners of the mouth of Bos taurus taurus with normal and improper nutrition at the electronic microscopic level indicate adaptive mechanisms for changes caused by the specific nutrition of ruminants and indicate the negative consequences of feeding animals with food unusual for ruminants, leading to deformation of the morphofunctional structures of the oral mucosa cavity and complete suppression of its symbiocenoses. This is a demonstration of 
the limited adaptive capabilities of the morphological structures of the oral cavity during extreme changes in the diet.

The study results clearly showed the correlation of the morphological features of the functional structures of the oral cavity and their symbiocenoses in ruminant ungulates and their dependence on the specifics of their content and nutrition. The data obtained can be used as a criterion for predicting the well-being of populations based on the characteristics of their nutrition and in the scientific development of standards for the correct feeding of domestic and wild species of ruminant ungulates under various conditions of their maintenance.

The author expresses his deep gratitude to the personnel of the KEM IPEE RAS: A.N. Nekrasov and A.N. Neretina for help in conducting electron microscopic studies. This paper was carried out using the equipment of the Center for Collective Use Instrumental Methods in Ecology at Institute of Ecology and Evolution named after A.N. Severtsov, RAS.

\section{References}

1. R.E. Hungate, Proceed. XIII Internat Congress of Nutrition (John Libbey, 1985)

2. P.H. Smith, R.E. Hungate, J. Bacteriol, 75, 6 (1968)

3. W. Pérez, H.E. König, H. Jerbi, M. Clauss, Vertebrate Zoology (2016)

4. R.R. Hofmann, Bul. Royal Soc. of New Zealand, 22 (1985)

5. R.R. Hofmann, Oecologia (1989)

6. M. Clauss, R.R. Hofmann, J. Hummel, J. Adamczewski, K. Nygren, C. Pitra, S. Reese, J. Zool., 270 (2006)

7. M. Clauss, T. Kaiser, J. Hummel, The ecology of browsing and grazing (2008)

8. K. Nygren, R.R. Hofmann, Alces, 26 (1990)

9. P.J. Van Soest, OB Books, Corvallis (1982)

10. R.M. Khatsaeva, Zoological Journal, 83, 12 (2004)

11. R.M. Khatsaeva, PH. D dissertation Dept. Biol. Sciences (2005)

12. D. Codron, M. Clauss, Canadian Journal of Zoology, 88 (2010)

13. C.H. Janis, Evolution, 30 (1976)

14. D. Archer, G. Sanson, Journal of Zoology, 257 (2002)

15. J.J.N Heywood, Journal of Zoology, 281 (2010)

16. T.M. Kaiser, J. Fickel, W.J. Streich, J. Hummel, M. Clauss, Journal of Zoology, 258 (2010)

17. J. Damuth, C.M. Janis, Biological Reviews, 86 (2011) 